Research Article

\title{
Principal Primitive Ideals in Quadratic Orders and Pell's Equations
}

\begin{abstract}
Ahmad Issa $(i)$ and Hasan Sankari
Department of Mathematics, Tishreen University, Latakia, Syria

Correspondence should be addressed to Ahmad Issa; ahmad.issa@tishreen.edu.sy

Received 31 August 2021; Revised 16 September 2021; Accepted 18 September 2021; Published 8 November 2021

Academic Editor: Marco Fontana

Copyright (C) 2021 Ahmad Issa and Hasan Sankari. This is an open access article distributed under the Creative Commons Attribution License, which permits unrestricted use, distribution, and reproduction in any medium, provided the original work is properly cited.

In this paper, we introduce a method of determining whether the primitive ideal is principal in a real quadratic order, depending on the solvability of Pell's equation.
\end{abstract}

\section{Introduction}

Let $d>1$ be square-free integer and $n$ a nonzero integer. Then, the equation $x^{2}-d y^{2}=n$ is known as Generalized Pell's equation. This equation has been studied by many authors (cf. $[2,4,6-8])$. In $[1,3,5]$, this equation has been applied to study the class numbers of real cyclotomic fields.

In this work, we give necessary and sufficient condition to solve $\left|x^{2}-d y^{2}\right|=n$, depending on the ideals in orders of the real quadratic fields. We also introduce the formula of the opposite ideal for every solution of this equation, in special cases of $d$ and $n$.

\section{Preliminaries}

A nonsquare integer $\Delta \in \mathbb{Z}$ is called a quadratic discriminant if $\Delta \equiv 0$ or $1(\bmod 4)$, we set $\omega_{\Delta}=\left(\sigma_{\Delta}+\sqrt{\Delta}\right) / 2$, where $\sigma_{\Delta}=$ $\left\{\begin{array}{ll}0, & \text { if } \Delta \equiv 0(\bmod 4) \\ 1, & \text { if } \Delta \equiv 1(\bmod 4)\end{array}\right.$ and
$\mathcal{O}_{\Delta}=\left[1, \omega_{\Delta}\right]=\mathbb{Z}\left[\omega_{\Delta}\right]=\left\{\frac{u+v \sqrt{\Delta}}{2} \mid u, v \in \mathbb{Z}, \quad u \equiv v \Delta(\bmod 2)\right\}$.

We call $\omega_{\Delta}$ the basic number and $\mathscr{O}_{\Delta}$ the order of the quadratic discriminant $\Delta$.
Now, we investigate ideals. Let $\Delta$ be a quadratic discriminant. An ideal I $\subset \mathcal{O}_{\Delta}$ is called $\mathcal{O}_{\Delta}$-primitive if $e^{-1} \mathrm{I} \nsubseteq \mathcal{O}_{\Delta}$ for all $e \in \mathbb{N}_{\geq 2}$. For a nonzero ideal I of $\mathcal{O}_{\Delta}$, we call $N(\mathrm{I})=$ $\left(\mathcal{O}_{\Delta}: \mathrm{I}\right)$ the absolute norm of I. If $\alpha$ is a nonzero of $\mathcal{O}_{\Delta}$, then $N(\langle\alpha\rangle)=|N(\alpha)|$.

\section{Main Results}

In this section, we will prove our main results. As a start, we record the following theorem.

Theorem 1. Let $\Delta=4 d \in \mathbb{N}$ be a quadratic discriminant. Suppose that $c=1$ if $d \equiv 3(\bmod 4)$, and $c=2$ if $d$ $\equiv 0(\bmod 8), d=c^{2} a b$, where $a, b \in \mathbb{N}$, and $a$ is odd and square-free. Then,

(1) $\mathrm{I}=\langle 2 c a, c a+\sqrt{d}\rangle$ is an $\mathcal{O}_{\Delta}$-primitive ideal satisfying $N(\mathrm{I})=2 \mathrm{ca}$. In addition, the following assertions are equivalent:

(a) I is a principal ideal of $\mathcal{O}_{\Delta}$.

(b) There exist $x, y \in \mathbb{Z}$ such that $\left|x^{2}-d y^{2}\right|=2 c a$ and $\operatorname{gcd}(c,(x / c) y)=1$.

(2) Let $x, y \in \mathbb{Z}$ be such that $\left|x^{2}-d y^{2}\right|=2 c a$ and $g c d$ $(c,(x / c) y)=1$. Then, 


$$
\mathrm{I}=\langle x+y \sqrt{d}\rangle \text {. }
$$

Proof (1). I is an ideal of $\mathcal{O}_{\Delta}$ satisfying $N(\mathrm{I})=2 \mathrm{ca}$. Let $e^{-1} \mathrm{I} \subset \mathcal{O}_{\Delta}$ with $e \in \mathbb{N}$ which implies $e=1$. Therefore, $\mathrm{I}$ is an $\widehat{O}_{\Delta}$-primitive ideal.

(a) $\Longrightarrow$ (b) Let I be principal. Then, there exists $\alpha=\left(x_{1}+y_{1} \sqrt{\Delta}\right) / 2 \in \mathcal{O}_{\Delta}$, where $\mathrm{I}=\langle\alpha\rangle=\alpha \mathcal{O}_{\Delta}$. We set $u=\left(x_{1} / 2\right), v=y_{1}$. Then, $\mathrm{I}=\langle u+v \sqrt{d}\rangle$. Since $\mathrm{I}$ is an $\mathcal{O}_{\Delta}$-primitive ideal, gcd $(u, v)=1$.

If $c=1, \mathrm{I}=\langle 2 a, a+\sqrt{d}\rangle=\langle u+v \sqrt{d}\rangle$, then

$$
2 a=\mathrm{N}(\mathrm{I})=|N(u+v \sqrt{d})|=\left|u^{2}-d v^{2}\right| \text {. }
$$

On the other hand, if $c=2, \quad \mathrm{I}=\langle 4 a, 2 a+\sqrt{d}\rangle$ $=\langle u+v \sqrt{d}\rangle$, since $\alpha \in \mathrm{I}$, there exist $m, Y \in \mathbb{Z}$ such that

$$
u+v \sqrt{d}=4 a m+(2 a+\sqrt{d}) Y=2 a(2 m+Y)+Y \sqrt{d} \text {. }
$$

If $X=2 m+Y$, then $u=2 a X$ and $v=Y$, so $\mathrm{I}=(2 a X+$ $Y \sqrt{d}) \mathcal{O}_{\Delta}$ and $\operatorname{gcd}(2, a X Y)=1$. On the other hand, $4 a=\mathrm{N}(\mathrm{I})=|\mathrm{N}(2 a X+Y \sqrt{d})|=\left|4 a^{2} X^{2}-d Y^{2}\right|$. Let $x=$ $2 a X$ and $y=Y$; hence, $\left|x^{2}-d y^{2}\right|=4 a$ and $(2,(x / 2) y)=1$.

$$
(b) \Longrightarrow(a) \text {. }
$$

If $c=1$, let $x, y \in \mathbb{Z}$ such that $\left|x^{2}-d y^{2}\right|=2 a$, which implies $x=a x_{1}$, for some $x_{1} \in \mathbb{Z}$ and $\operatorname{gcd}(2, x y)=1$; then, $\operatorname{gcd}\left(2, x_{1}\right)=1$ and $x_{1}-y=2 w$, for some $w \in \mathbb{Z}$. Now, we obtain $x+y \sqrt{d}=2 a w+y(a+\sqrt{d}) \in \mathrm{I}$; hence, $\langle x+y \sqrt{d}\rangle$ $C \mathrm{I}$, and equality holds since

$$
N(\langle x+y \sqrt{d}\rangle)=|N(x+y \sqrt{d})|=\left|x^{2}-d y^{2}\right|=2 a=N(I) .
$$

If $c=2$, let $x, y \in \mathbb{Z}$ such that $\left|x^{2}-d y^{2}\right|=4 a$ and $(2,(x / 2) y)=1$; then, $\left|(x / 2)^{2}-a b y^{2}\right|=a$, which implies $(x / 2)=a x_{1}$, for some $x_{1} \in \mathbb{Z}$; then, $x_{1}$ is odd and $x_{1}-y=2 w$, for some $w \in \mathbb{Z}$ Now, we obtain $x+y \sqrt{d}=4 a w+y(2 a+\sqrt{d}) \in \mathrm{I}$. Hence, $\langle x+y \sqrt{d}\rangle \subset \mathrm{I}$, and equality holds since

$$
N(\langle x+y \sqrt{d}\rangle)=|\mathrm{N}(x+y \sqrt{d})|=4 a=N(I) .
$$

(2) By (b) $\Longrightarrow$ (a). Now, we will be devoted to numerical examples illustrating Theorem 1.

Example 1. Let $\Delta=364=4 d$ and $d=91=7 \times 13=a b \equiv 3$ $(\bmod 4)$. Since $(105,11)$ is the solution of $x^{2}-91 y^{2}=14$; hence, $\langle 14,7+\sqrt{d}\rangle=\langle 105+11 \sqrt{d}\rangle$.

Example 2. Let $\Delta=1248=4 d$ and $d=312=4 \times 3 \times 26=$ $4 a b \equiv 0(\bmod 8)$. Since $(18,1)$ is the solution of $x^{2}-312 y^{2}=12$; hence, $\langle 12,6+\sqrt{d}\rangle=\langle 18+\sqrt{d}\rangle$.

Theorem 2. Let $\Delta=4 d \in \mathbb{N}$ be a quadratic discriminant. Suppose that $d=4 a b$, where $a, b \in \mathbb{N}$, and $a$ is square-free. Then,
(1) $I=\langle a, \sqrt{d}\rangle$ is an $\mathcal{O}_{\Delta}$-primitive ideal satisfying $N(\mathrm{I})=a$. In addition, the following assertions are equivalent:

(a) I is a principal ideal of $\mathcal{O}_{\Delta}$.

(b) There exist $x, y \in \mathbb{Z}$ such that $\left|x^{2}-d y^{2}\right|=4 a$ and $\operatorname{gcd}(x, y)=2$.

(2) Let $x, y \in \mathbb{Z}$ be such that $\left|x^{2}-d y^{2}\right|=4 a$ and $g c d$ $(x, y)=2$.Then,

$$
\mathrm{I}=\left\langle\frac{x}{2}+\frac{y}{2} \sqrt{d}\right\rangle
$$

Proof (1). I is an ideal of $\mathcal{O}_{\Delta}$ satisfying $N(\mathrm{I})=a$. Let $e^{-1} \mathrm{I} \subset \mathcal{O}_{\Delta}$ with $e \in \mathbb{N}$ which implies $e=1$. Therefore, I is $\mathcal{O}_{\Delta}$-primitive.

(a) $\Longrightarrow$ (b) Let I be principal. Then, there exists $\alpha=\left(x_{1}+y_{1} \sqrt{\Delta}\right) / 2 \in \mathcal{O}_{\Delta}$, where $\mathrm{I}=\langle\alpha\rangle=\alpha \mathcal{O}_{\Delta}$. We set $u=\left(x_{1} / 2\right), v=y_{1}$. Then, $\mathrm{I}=(\langle u+v \sqrt{d}\rangle)$. Since $\mathrm{I}$ is an $\mathcal{O}_{\Delta}$-primitive ideal, gcd $(u, v)=1$.

On the other hand, $a=N(\mathrm{I})=|N(u+v \sqrt{d})|$ $=\left|u^{2}-d v^{2}\right|$. Let $x=2 u$ and $y=2 v$; then, $\left|x^{2}-d y^{2}\right|=4 a$ and $\operatorname{gcd}(x, y)=2$.

(2) (b) $\Longrightarrow$ (a) Let $x, y \in \mathbb{Z}$ such that $\left|x^{2}-d y^{2}\right|=4 a$ and $\operatorname{gcd}(x, y)=2$. Then, $\left|(x / 2)^{2}-a b y^{2}\right|=a$, which implies $(x / 2)=a x_{1}$, for some $x_{1} \in \mathbb{Z} . \quad(x / 2)+(y / 2) \sqrt{d}$ $=a x_{1}+(y / 2) \sqrt{d} \in \mathrm{I}$. Hence, $((x / 2)+(y / 2) \sqrt{d}) \mathcal{O}_{\Delta} \subset \mathrm{I}$, and equality holds since

$$
N\left(\left(\frac{x}{2}+\frac{y}{2} \sqrt{d}\right) \mathcal{O}_{\Delta}\right)=\left|\mathrm{N}\left(\frac{x}{2}+\frac{y}{2} \sqrt{d}\right)\right|=\left|\frac{x^{2}-d y^{2}}{4}\right|=a=N(\mathrm{I}) .
$$

(2) $\mathrm{By}(\mathrm{b}) \Longrightarrow$ (a). Now, we will be devoted to numerical examples illustrating Theorem 2 .

Example 3. Let $\Delta=1488=4 d$ and $d=372=4 \times 3 \times 31$ $=4 a b$. Since $(270,14)$ is the solution of $x^{2}-372 y^{2}=-12$; hence, $\langle 3, \sqrt{d}\rangle=\langle 135+7 \sqrt{d}\rangle$.

\section{Data Availability}

No data were used to support this study.

\section{Conflicts of Interest}

The authors declare that they have no conflicts of interest.

\section{Acknowledgments}

This work was supported by Tishreen University.

\section{References}

[1] S. D. Lang, "Note on the class-number of the maximal real subfield of a cyclomatic field," Journal für die Reine und Angewandte Mathematik, vol. 290, pp. 70-72, 1977. 
[2] F. Halter-Koch, "Diophantine equations of Pellian type," Journal of Number Theory, vol. 131, no. 9, pp. 1597-1615, 2011.

[3] A. Hoque and H. K. Saikia, "On the class-number of the maximal real subfield of a cyclotomic field," Quaestiones Mathematicae, vol. 39, no. 7, pp. 889-894, 2016.

[4] A. Hoque and K. Chakraborty, "Pell-type equations and class number of the maximal real subfield of a cyclotomic field," The Ramanujan Journal, vol. 46, no. 3, pp. 727-742, 2018.

[5] A. Hoque and S. Kotyada, "Class number one problem for the real quadratic fields $\$ \$\{\{\backslash$ mathbb $\{\mathrm{Q}\}(\{\backslash \operatorname{sqrt}\{\mathrm{m} 2+2 \mathrm{r}\}\})\}\} \$ \$$," Archiv der Mathematik, vol. 116, no. 1, pp. 33-36, 2021.

[6] A. Issa and H. Sankari, "Some criteria for class numbers to be non-one," Journal of Mathematics, vol. 2020, Article ID 5672983, 5 pages, 2020.

[7] P. Kaplan and K. S. Williams, "'Pell's equations X2 - mY2 = -1, -4 and continued fractions," Journal of Number Theory, vol. 23, no. 2, pp. 169-182, 1986.

[8] H. Yokoi, "Solvability of the diophantine equation $\mathrm{x} 2-\mathrm{Dy} 2= \pm$ 2 and new invariants for real quadratic fields," Nagoya Mathematical Journal, vol. 134, pp. 137-149, 1994. 